\title{
Smartphone-based Optical Fiber Speckle Spectrometer
}

\author{
Henry Tan ${ }^{1}$, Jasper J. Cadusch ${ }^{2}$, and Kenneth B. Crozier ${ }^{1,2}$ \\ ${ }^{1}$ School of Physics, University of Melbourne, Victoria, 3010, Australia \\ ${ }^{2}$ Department of Electrical and Electronic Engineering, University of Melbourne, Victoria, 3010, Australia \\ Corresponding Author e-mail address: kcrozier@unimelb.edu.au
}

\begin{abstract}
We demonstrate a spectrometer that uses a smartphone to image the speckle pattern produced by a multimode optical fiber. An algorithm uses the measured pattern and a calibration library to determine the visible-wavelength input spectrum. (C) 2020 The Author(s)
\end{abstract}

\section{Introduction}

The smartphone represents a powerful platform for scientific instruments. Notable developments of this approach include microscopes $[1,2]$ and point-of-care medical diagnostics [3,4]. Smartphone-based spectrometers based on diffraction gratings have also been demonstrated $[5,6]$. For grating-based spectrometers, however, resolution scales inversely with grating size. This motivates the development of smartphone-based spectrometers based on approaches other than gratings, to enable high resolution even from small platforms. This is the topic of this study. We demonstrate, for the first time to the best of our knowledge, a spectrometer that uses a smartphone to image the speckle pattern produced by a multimode optical fiber. The speckle phenomenon is very promising for spectroscopy. Redding et al demonstrated spectroscopy at $\lambda \sim 1500 \mathrm{~nm}$ with a resolution of $1 \mathrm{pm}$ [7]. Metzger et al demonstrated a wavemeter at $\lambda \sim 780 \mathrm{~nm}$ with a resolution of $0.3 \mathrm{fm}$ [8]. In those works, speckle patterns produced by multimode optical fibers [7] and integrating spheres [8] were imaged with benchtop systems (e.g. optical microscopes). Here, we show that a smartphone can be used for speckle spectroscopy, reconstructing various visible-wavelength spectra.

\section{Experimental Setup}

The experimental setup is shown in Fig. 1a. Light from a supercontinuum source equipped with a tuneable filter (NKT Photonics) impinges upon a beam splitter. The reflected beam is input to a reference spectrometer (Ocean Optics QEPro), allowing source spectra during the calibration step to be recorded (Fig. 1b). The transmitted beam is focused by a fiber coupler into an optical fiber. The latter consists of polarization-maintaining single-mode fiber (PMF, $1 \mathrm{~m}$ long) spliced to multimode fiber (MMF, $6 \mathrm{~cm}$ long, core diameter $=105 \mu \mathrm{m}, \mathrm{NA}=0.22$ ). An image of the MMF output is obtained with a microscope that consists of a smartphone (LG Nexus $5 \mathrm{X}$ ) to which a lens has been added. The latter is termed a "reversed lens" [1] to denote the fact that it consists of a camera module that is identical to the camera module present in the smartphone, except that it is placed in the reverse orientation. This effectively turns the smartphone into a microscope with unity magnification [1], allowing it to capture speckle patterns (Fig. 1c).

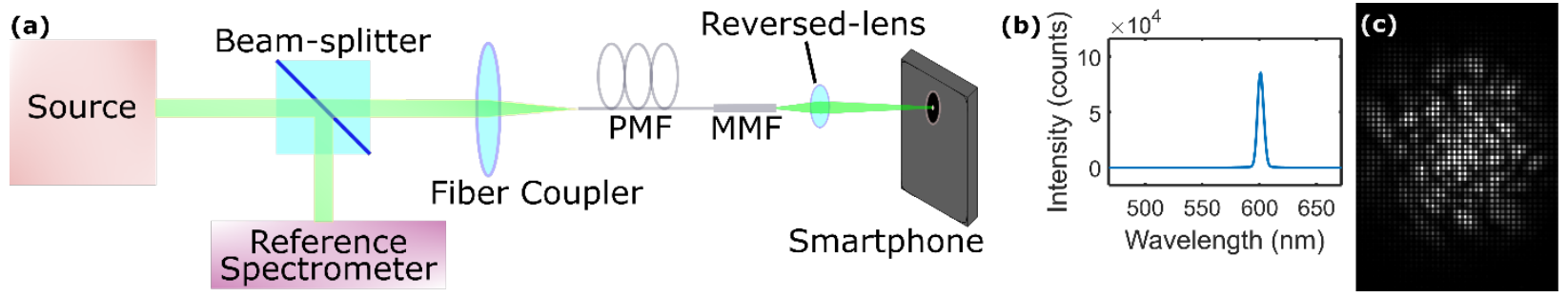

Fig 1. (a) Schematic of experimental setup. (b) Example $600 \mathrm{~nm}$ signal by source. (c) Resultant speckle pattern imaged (107 by 75 pixel).

To calibrate our spectrometer, we sweep the source from $\lambda=470 \mathrm{~nm}$ to $670 \mathrm{~nm}$ with a step size of $2 \mathrm{~nm}$, imaging the speckle pattern at each step with the smartphone and measuring the spectrum with the reference spectrometer. This creates a reference library of 101 pairs of calibration patterns and spectra.

The conversion of the input spectrum into a speckle pattern by the MMF is modeled by $I=T \cdot S_{I}$. The speckle pattern (whose spectrum is to be determined) is stored as a vector $I$, and the transmission matrix $T$ stores all the calibration patterns column-wise. Determination of $S_{I}$ is an inverse problem that cannot be solved exactly, and we instead use a least-squares non-negative regression algorithm (in MATLAB) to find an approximation $S_{I}{ }^{\prime}$. With $S_{I}{ }^{\prime}$ known, the reconstructed spectrum $S^{\prime}$ is recovered via $A \cdot S_{I}^{\prime}=S^{\prime}$, where $A$ stores calibration spectra, analogous to $T$.

\section{Results and Discussion}

In Fig. 2a, we test our smartphone spectrometer by reconstructing 19 different single peak spectra. The average spectral reconstruction error $\mu$ (standard deviation between reconstructed spectra and those measured directly with 
reference spectrometer, [7]) of the 19 tests is 0.40 . In Fig. $2 \mathrm{~b}$ and 2c, we single out the best performing test. The $S_{I}{ }^{\prime}$ in Fig. $2 \mathrm{~b}$ identifies the correct speckle pattern with minimal cross-talk. The spectral reconstruction is shown in Fig. $2 \mathrm{c}$, with $\mu=0.24$. The observed speckle pattern (false-color) associated with a broad spectrum is shown in Fig. 3a. It can be seen that the speckle pattern (Fig. 3b) associated with our reconstruction (Fig. 3c) appears very similar to the measured speckle (Fig. 3a). The spectral reconstruction is thus in good agreement with the reference spectrum (Fig. $3 \mathrm{~d}, \mu=0.52)$. Fig. 4 demonstrates that our smartphone spectrometer can distinguish between metameric signals. Metamerism is the phenomenon where different spectra appear the same color to human eyes or color cameras.
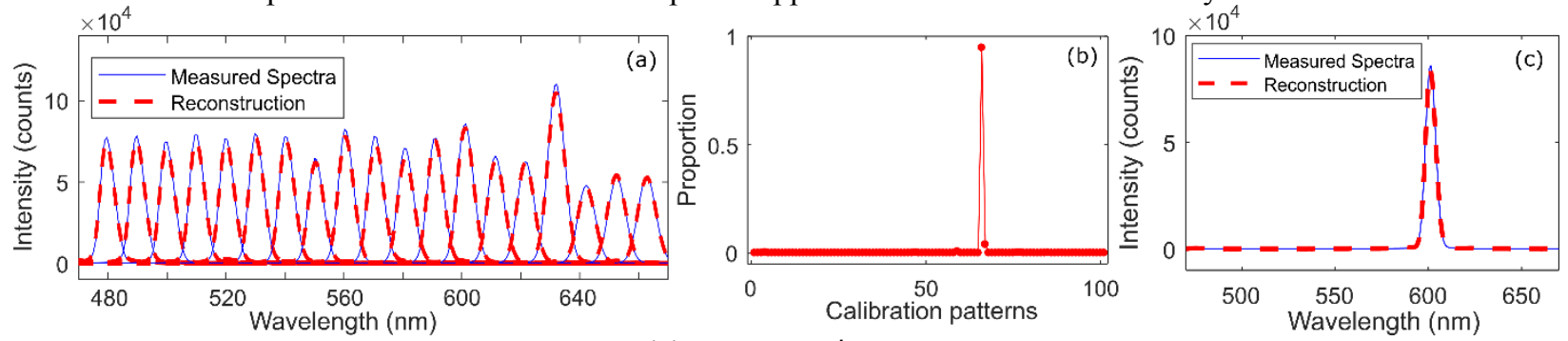

Fig. 2. (a) Reconstruction of 19 other single-peak spectra: $\langle\mu\rangle=0.40$. (b) $S_{I}{ }^{\prime}$ of $600 \mathrm{~nm}$ signal. (c) Resultant spectral reconstruction: $\mu=0.24$.
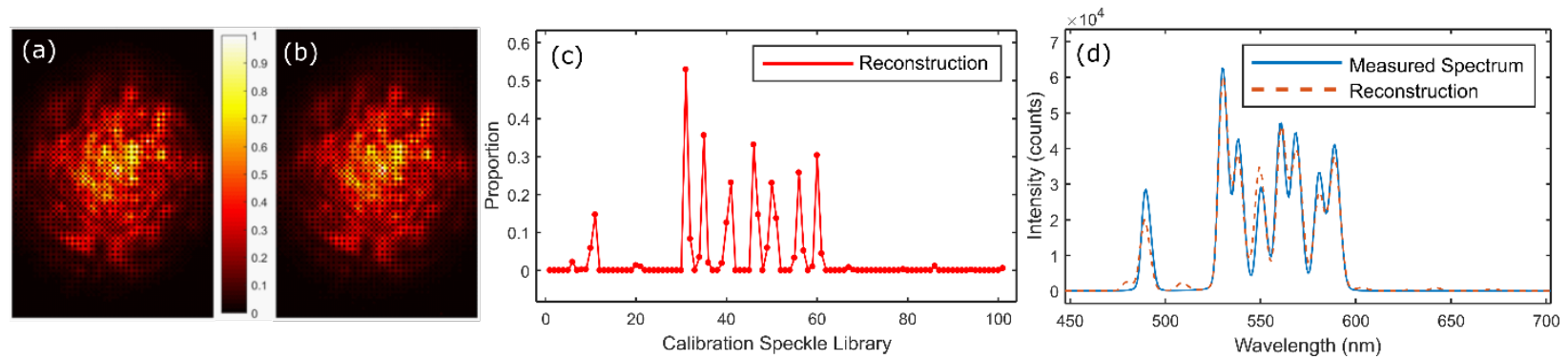

Fig. 3. (a) Imaged speckle pattern (false-color). (b) Reconstructed pattern. (c) $S_{I}{ }^{\prime}$. (d) Resultant spectral reconstruction: $\mu=0.52$
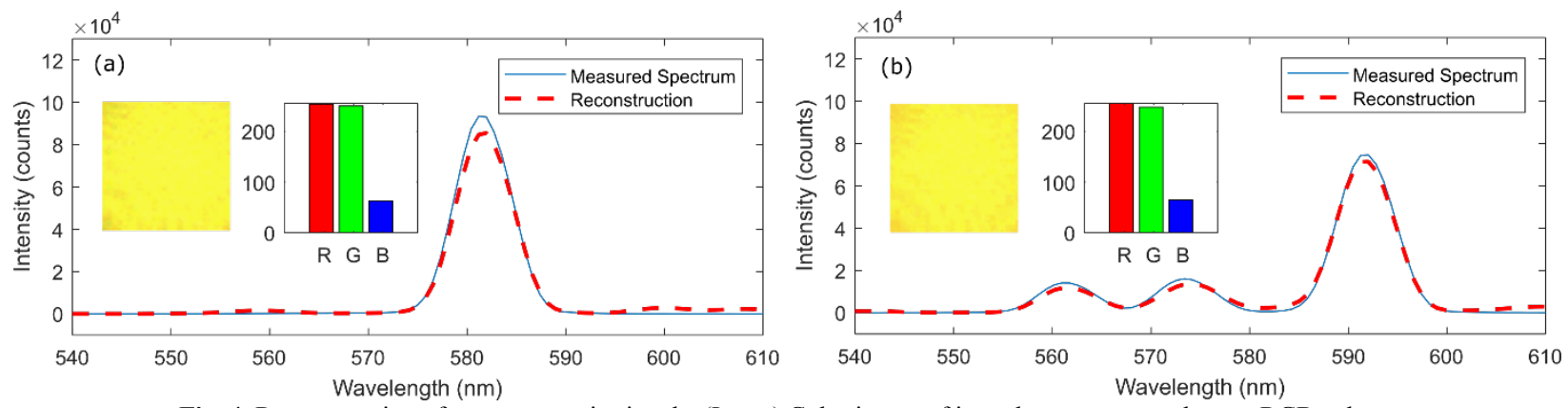

Fig. 4. Reconstruction of two metameric signals. (Insets) Color image of input beam center and mean RGB values.

In summary, we demonstrate a visible-wavelength spectrometer that uses a smartphone to image the speckle pattern produced by a multimode optical fiber. Future work may involve calibrating the system with a source that has narrower linewidth than that employed in this work $(\sim 5.5 \mathrm{~nm}$ full-width at half maximum) to improve resolution still further.

1. N. A. Switz, M. V. D’Ambrosio, and D. A. Fletcher, "Low-cost mobile phone microscopy with a reversed mobile phone camera lens," PLoS One 9, (2014)

2. Y. Rivenson et al., "Deep learning enhanced mobile-phone microscopy," ACS Photon. 5, 2354 (2018)

3. T. Ghonge, H. C. Koydemir, E. Valera, et al, "Smartphone-imaged microfluidic biochip for measuring CD64 expression from whole blood," Analyst 144, 3925 (2019)

4. H-A Joung, Z.S. Ballard, J. Wu, D.K. Tseng, et al, "Point-of-care serodiagnostic test for early-stage Lyme disease using a multiplexed paperbased immunoassay and machine learning," medRxiv 19009423 (2019)

5. H. Yu, Y. Tan, and B. T. Cunningham, "Smartphone fluorescence spectroscopy," Anal. Chem. 86, 8805-8813 (2014)

6. M. A. Hossain, J. Canning, Z. Yu, et al, "Time-resolved and temperature tuneable measurements of fluorescent intensity using a smartphone fluorimeter," Analyst 142, 1953 (2017)

7. B. Redding, M. Alam, M. Seifert, and H. Cao, "High-resolution and broadband all-fiber spectrometers," Optica 1, 175-180 (2014) 8. N. Metzger, N., R. Spesyvtseva, G. Bruce, et al, "Harnessing speckle for a sub-femtometre resolved broadband wavemeter and laser stabilization," Nat Commun 8, 15610 (2017) 


\section{University Library}

\section{- M M I E E R VA A gateway to Melbourne's research publications}

Minerva Access is the Institutional Repository of The University of Melbourne

Author/s:

Tan, H;Cadusch, JJ;Li, B;Crozier, KB

Title:

Smartphone-based Optical Fiber Speckle Spectrometer

Date:

2020-01-01

Citation:

Tan, H., Cadusch, J. J., Li, B. \& Crozier, K. B. (2020). Smartphone-based Optical Fiber Speckle Spectrometer. 2020 CONFERENCE ON LASERS AND ELECTRO-OPTICS (CLEO), 2020-May, IEEE. https://doi.org/10.1364/cleo_si.2020.sm2I.2.

Persistent Link:

http://hdl.handle.net/11343/294868 